\title{
Propagation and Reflection of Gas Waves in a Close Tube
}

\author{
S. T. Chen*, D. P. Hu, Z. Z. Chen, C. Zhu, R. J. Liu, Y. Q. Dai \\ Institute of Chemical Engineering, Dalian University of Technology, Dalian, Liaoning 116012, China \\ Email: dutchenshengtao@sina.com
}

\begin{abstract}
The gas and shock wave's motion in a receiving tube is investigated numerically and experimentally. The results show that, the velocity of the contact face rises rapidly as gas is injected into the receiving tube, and drops sharply after a steady propagation. However, the velocity of the shock wave in the tube is almost linear and the shock wave can be reflected at the close end of the receiving tube. The shock wave and the injecting wave propagate in the different direction. When the reflected wave sweeps, it can heat the gas about $20 \mathrm{~K}$ under the pressure ratio of 2 . If the exhausted gas is used to refrigeration, the reflected wave has negative effect. With increasing of inlet pressure, the velocity of the shock wave and steady velocity of contact face also increase. There is obvious thermal effect as the shock wave sweeps the gas. The reflected shock wave can heat the exhausting gas in the open end. The exhausting gas' tempcrature for low pressure ratio is higher than the temperature for high pressure ratio. But as the pressure ratio rises to a value, the temperature drop little. The result shows that the numerical value is a little higher than the experimental value, especially under lower pressure ratio. The reason may lie in the operation condition and the heat exchange. Under lower pressure ratio, the shock wave can heat the gas in the closed end and the heat can easily be transferred to the open end. As a whole, the experimental results are consistent with the numerical simulation.
\end{abstract}
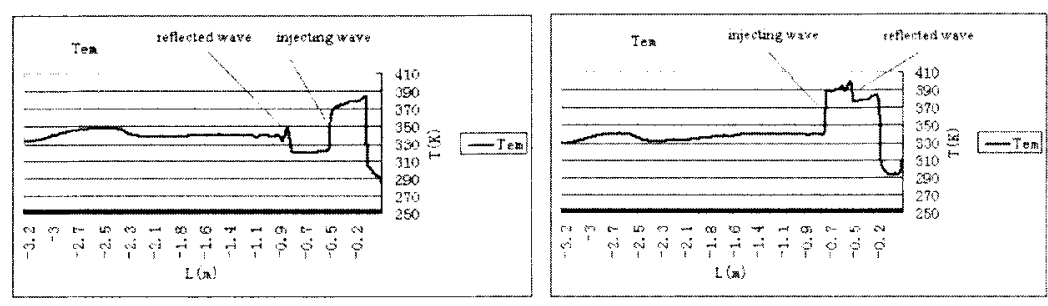

Figure: Shock wave's reflection

\section{REFERENCES}

1. Hatazawa. Oscillatory flow in the thermoacoustic sound wave generator. Journal of Thermal Science, 2006; 15(1)

2. Speziale C G, Thangam S. Analysis of an RNG based turbulence model for separated flows. Int $J$ Engng Sci, 1992; 10: 1397-1388

3. Fang Y, Zhu C. Experimental study of gas wave refrigeration. In: The 1st International Shock Wave Proceedings, Sendai, Japan 1991; 2: 1335-1338 